International Journal of Wireless \& Mobile Networks (IJWMN) Vol. 3, No. 5, October 2011

\title{
Performance Evaluation of AODV Routing Protocol in Cognitive Radio Ad-hoc Network
}

\author{
Prof.Shubhangi Mahamuni ${ }^{1}$ \\ Dr.Vivekanand Mishra (Senior Member IEEE) ${ }^{2}$ \\ Dr.Vijay M.Wadhai ${ }^{3}$ \\ ${ }^{1}$ Assist. Prof, Dept.of E\&TC, MAE, Alandi (D), Pune,MS. \\ Shubhangimahamuni 4 @gmail.com \\ ${ }^{2}$ Associate Professor, Dept.of Electronics, SVNIT, Surat,Gujrat. \\ vive2009@gmail.com \\ ${ }^{3}$ Principal, MITCOE, Kothrud,Pune, MS \\ wadhai.vijay@gmail.com
}

\begin{abstract}
-
A cognitive radio is designed for the utilisation of unused frequency. The transmission opportunity of a cognitive node is not guaranteed due to the presence of primary users (PUs).To better characterize the unique features of cognitive radio networks, we propose new routing metrics, including Routing for CRNs using IEEE 802.11 which are the official standards for wireless communication. Routing protocols, for network without infrastructures, have to be developed. These protocols determine how messages can be forwarded, from a source node to a destination node which is out of the range of the former, using other mobile nodes of the network. Routing, which includes for example maintenance and discovery of routes, is one of the very challenging areas in communication. Numerous simulations of routing protocols have been made using different simulators, such as ns-2.The impact of sensing time, route path and mobility in Ad-Hoc networks on connectivity and throughput tested.
\end{abstract}

Keywords: Ccognitive radio, CRAHNS (Cognitive Radio Adhoc Network), Spectrum sensing, Dynamic Spectrum Access, spectrum handoff.

\section{Introduction}

Cognitive Radio (CR) can be used as a solution to current unbalanced spectrum utilization. The cognitive ad hoc network can take advantage of dynamic spectrum access and spectrum diversity over wide spectrum. It could achieve higher network capacity compared to traditional ad hoc networks, thus supporting bandwidth-demanding applications. Mobile Ad- Hoc networking has gained an important part of the interest of researchers and become very popular these past few years, due to its potential and possibilities. These protocols determine how messages can be forwarded, from a source node to a destination node which is out of the range of the former, using other mobile nodes of the network. Routing, which includes for example maintenance and discovery of routes, is one of the very challenging areas in communication. Simulators though cannot take into account of all the factors that can come up in real life and performance and connectivity of mobile Ad-Hoc network depend and are limited also by such factors. Here we have tried routing protocol and end to end protocol to enhance throughput of a cognitive radio adhoc network . The impact of varying packet size, route length and mobility in Ad- Hoc networks on connectivity, Route Discovery Time and throughput is tested. In this paper we included an introduction to Ad-Hoc Mobile Networks and IEEE 802.11in part I. Part II consists routing in CRAHNs. Simulation flowchart, algorithm, results and software are described in chapter III. 
International Journal of Wireless \& Mobile Networks (IJWMN) Vol. 3, No. 5, October 2011

\section{A. Cognitive Radio Ad-Hoc Network}

Cognitive Radio Ad-Hoc Network (CRAHNs) $[1,2]$ is a new developed technology of wireless communication. The difference to traditional wireless networks is that there is no need for established infrastructure. Since there is no such infrastructure and therefore no preinstalled routers which can, for example, forward packets from one host to another, this task has to be taken over by participants, also called mobile nodes, of the network. Each of those nodes takes equal roles, what means that all of them can operate as a host and as a router. Traditional wireless networks are need some improvement due to some factors such as security, power control, transmission quality and bandwidth optimization. To solve problems like maintenance and discovery of routes and topological changes of the network is the challenge of Ad-Hoc Networking.

We have formed cognitive radio adhoc network by using user nodes and communication channels as shown in fig.1.

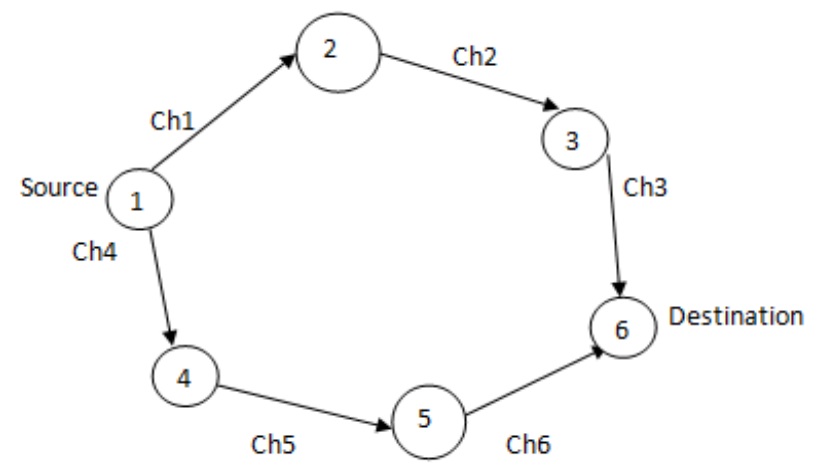

Fig.1 Cognitive Radio Ad-Hoc Network

\section{B. RELATED WORK}

A number of routing protocols have been proposed and implemented for CRAHNs in order to enhance the bandwidth utilization, higher throughputs, lesser overheads per packet, minimum consumption of energy and others. All these protocols have their own advantages and disadvantages under certain circumstances. The major requirements of a routing protocol was proposed by Tao Lin et al.[2] that includes minimum route acquisition delay, quick routing reconfiguration, loop-free routing, distributed routing approach. A review of Routing Protocols has been explained in detail by Changling Liu, Jorg Kaiser[3,4,5,6].Obviously, most of the routing protocols are qualitatively enabled. A lot of simulation studies were carried out in the paper [2] to review the quantitative properties of routing protocols. The idea of the Cognitive radio Adhoc network was explained by I. Akyildiz, W.Y. Lee, K.R. Chowdhury[1]. A number of extensive simulation studies on various CRAHNs routing protocols have been performed in terms of control, route discovery and route maintenance[7,8][9,10]. However, there is a severe lacking in implementation and operational experiences with existing CRAHNs routing protocols. The various types of mobility models were identified and evaluated by K.R. Choudhury, M.D. Felice et al. [17] because the mobility of a node will also affect the overall performance of the routing protocols. A framework for the ad hoc routing protocols was proposed by Tao Lin et al. [3] using Relay Node Set which would be helpful for comparing the various routing protocols like AODV, OLSR \& TBRPF [17]. Application of the standards like IEEE 802.11,TCP has explained in detail by M. Gandetto, C. Regazzoni and Goff, J. Moronski et al. $[13,14]$. The performance of the routing protocols AODV by considering the metrics of packet delivery ratio, control traffic overhead and route length by using NS2 simulator [18][19][20][21]. 
International Journal of Wireless \& Mobile Networks (IJWMN) Vol. 3, No. 5, October 2011

\section{II.AODV Protocol and Routing}

\section{A.AODV Protocol:}

AODV protocol is a routing protocol in a reactive routing protocol. Routing is only when needed. Fig.1 1shows message transmission using AODV protocol. Routing in a Cognitive Radio Adhoc Network (CRAHNs) is done with the goal of finding a short and optimized route from the source to the destination node. An advantage is that smaller bandwidth is needed for maintaining routing tables, and disadvantage is it will create non negligible delay, since before using the route for a specific communication, it has to be determined. When a source has to transmit to an unknown destination sends RREQ route request for the destination [3,4]. If the receiving node has not received RREQ,it is not the destination node and does not have the current route to the destination.RREP of used for hopping purpose from the souse towards destination, it generates route reply(RREP).for the creation of routes between the nodes RREP is used. As shown in fig.2 $\mathrm{S}$ is the source and $\mathrm{D}$ is the destination used for the transmission of simple message Hello towards destination $\mathrm{D}$.

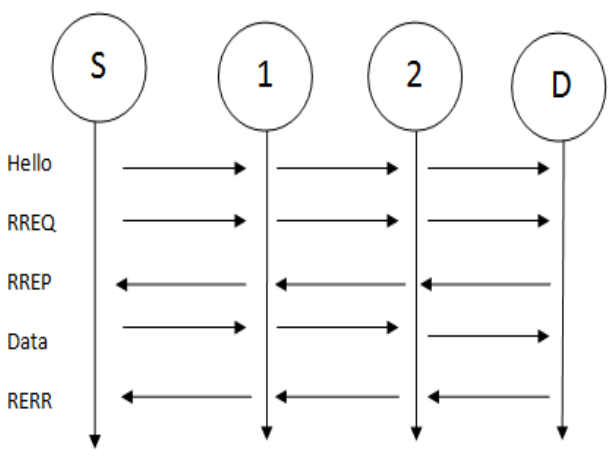

Fig.2 AODV Protocol Messaging

When a route is needed and it is not already known by a node it sends a Route Request (RREQ) message to its neighbours. Those forward the message until it reaches the destination node. Each intermediate node updates the RREQ with its address. When a node sends a RREQ message it attaches to it a request ID. The request ID and the IP address of the node form a unique identifier. This is done in order to prevent that an intermediate node which receives twice the same RREQ message forwards it twice. When the destination receives the RREQ it sends a Route Reply (RREP) message back to the source node by reversing the hop sequence recorded in the RREQ. When the source receives the RREP it can start communicating with the destination, by including the whole route in the header of each to be sent message.

\section{B. IEEE 802.11}

The scope of IEEE 802.11 [5] is to develop and maintain specifications for wireless connectivity for fixed, portable and moving stations within a local area. It defines over-the-air protocols necessary to support networking in a local area. This standard provides MAC and physical layer functionality.

IEEE 802.11 can be used in two different operating modes:

1. Infrastructure mode

2. Independent (Ad-Hoc) mode

At the MAC layer 802.11 uses both carrier sensing and virtual carrier sensing before sending data to avoid collision. Use of RTS and CTS is used for virtual carrier sensing. The network allocation vector (NAV) is used to perform virtual channel sensing for the indication that the channel is busy. 
International Journal of Wireless \& Mobile Networks (IJWMN) Vol. 3, No. 5, October 2011

ACK is used for the indication that the destination received data correctly. This way RTS-CTSData-ACK is called Distributed Coordination Function (DCF).

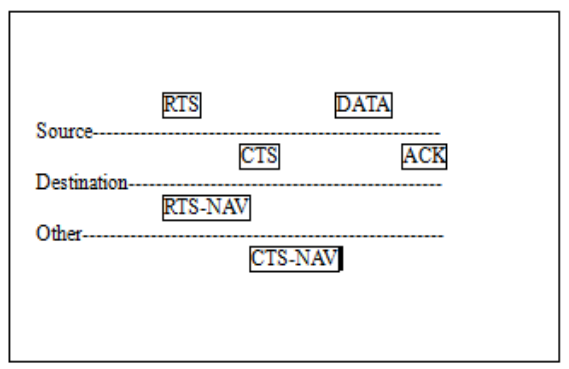

Fig.3 IEEE 802.11Disributed co-ordination Function

\section{III.Routing In a CRAHNs}

\section{A. Route Maintenance:}

When a route has been established, it is being maintained by the source node as long as the route is needed. Movements of nodes affect only the routes passing through this specific node and thus do not have global effects. If the source node moves while having an active session, and loses connectivity with the next hop of the route, it can rebroadcast an RREQ. If though an intermediate station loses connectivity with its next hop it initiates an Route Error (RERR) message and broadcasts it to its precursor nodes and marks the entry of the destination in the route table as invalid, by setting the distance to infinity[6,7]. The entry will only be discarded after a certain amount of time, since routing information may still be used for the transmission of message from source.Fig. 3 shows the shifting of path for transmission of data from different source and destination.

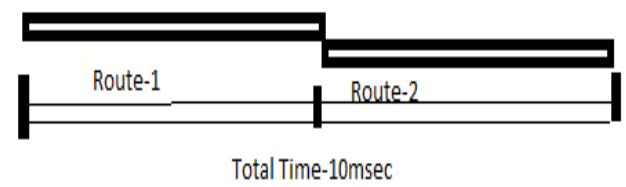

Fig.4.Channel Utilisation in CRAHNs

\section{B.Transport layer:}

Since TCP is the de facto transport protocol standard on Internet, it is crucial to estimate its ability in providing stable end-to-end communication over CRAHNs.In this set of experiments, the CRAHN environment is based on four channels (i.e. $N=4$ ) and constructed as follows We study the performance of TCP under different CRAHNs characteristics, e.g. the sensing time interval of CR users. The choice of the single-hop scenario can be motivated as follows. First, the single-hop scenario is simple enough to understand the impact of CRAHNs characteristics on the dynamics of TCP, while this might be difficult to investigate in multi-hop topologies. Second, the single-hop topology constitutes a base case, from the point of view of protocol performance. If we discover that a single parameter, e.g. the sensing time interval, has a strong impact on TCP performance, then this effect will be emphasized in a multi-hop environment by the presence of multiple intermediate nodes between the source and the destination CR users. Moreover, although very simple, the single-hop topology constitutes a realistic model for the evaluation of infrastructure-based CR networks, where the mobile CR users are attached to a fixed cognitive base station (BS).

We consider two metrics in the performance analysis: 
International Journal of Wireless \& Mobile Networks (IJWMN) Vol. 3, No. 5, October 2011

- TCP throughput: This is the end-to-end TCP throughput at the application layer, i.e. the amount of bits for seconds received by the upper layer FTP application at the destination node, without considering out-of-order, duplicated and TCP-ACK packets.

- TCP efficiency: This is an estimation of bandwidth resource utilization by TCP. It is defined as follows:

$$
\epsilon=\frac{T C P_{T H R}\left(t_{1}, t_{2}\right)}{\int_{t_{1}}^{t_{2}} C(t) \cdot \mathrm{d} t}, \quad 0 \leq \epsilon \leq 1
$$

(5)

where $\operatorname{TCP}_{\mathrm{THR}}(\mathrm{t} 1, \mathrm{t} 2)$ is the average TCP throughput computed over the measurement period from $\mathrm{t}$, and $C(t)$ is the available channel capacity at time $\mathrm{t}$. All the metrics described so far are measured at CR users since the focus here is to evaluate the impact of PU activity on the performance of the TCP protocol for secondary systems.

\section{C.Sensing Cycle Analysis Simulation Setup:}

In the sensing cycle analysis, we consider the basic single-hop topology, and we vary the sensing interval $(t s)$ and operation interval of CR users. All the primary bands have capacity equal to $5 \mathrm{Mb} / \mathrm{s}$.

\section{IIV. SIMULATION AND RESULTS:}

\section{A. Flowchart:}

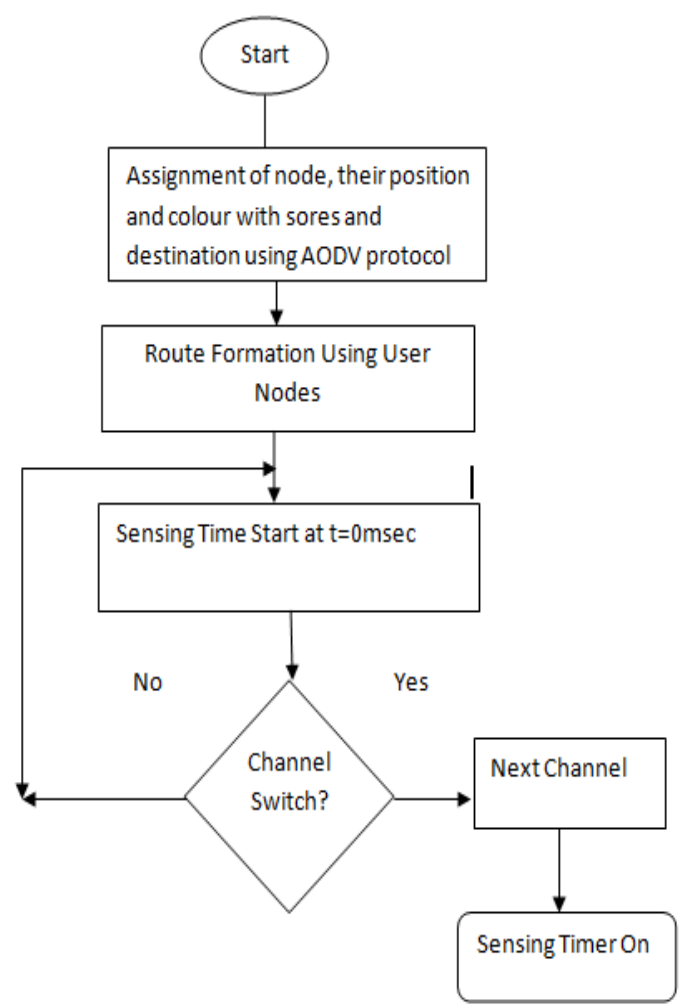

Fig.5. Flowchart for channel allocation and routing in Cognitive Radio Ah-doc Networks 
International Journal of Wireless \& Mobile Networks (IJWMN) Vol. 3, No. 5, October 2011

\section{B. Node Mobility:}

In CR ad-hoc networks, TCP-EFLN and A TCP react to the route disruption after it happens by an explicit notification in the form of the internet control message protocol (ICMP) message at the IP layer[8,9]. For CRAHNs, intermediate nodes may continue their periodic sensing if a route failure is detected at a further downstream node. In such cases, the route failure message is delayed at each hop that undertakes sensing and the source is informed much later. [10] and hence a predictive mobility model needs to be incorporated in the TCP rate control mechanism. Two broad approaches may be adopted in the design of transport layer protocols [11, 12].

(i) The standard TCP and UDP protocols may be adapted by making them channel aware and sensitive to PU activity.

(ii) Scenario and application specific protocols may be devised those tradeoffs the generality in implementation for optimum performance under known channel condition.

\section{Implementation of AODV Protocol}

Performance evolution of the AODV protocol is implemented on the Linux 2.4 Kernel with the following considerations.

\begin{tabular}{|l|l|}
\hline \multicolumn{1}{|c|}{ Parameter } & \multicolumn{1}{c|}{ Value } \\
\hline Channel Type & Channel/Wireless Channel \\
\hline propagation & Propagation/Two Ray Ground \\
\hline antenna Type & Antenna/Omni Antenna \\
\hline Network Layer & LL \\
\hline Queue & Queue/Drop Tail/PriQueue \\
\hline Queue Length & 200 \\
\hline Network Layer & Phy/WirelessPhy \\
\hline MAC protocol & Mac/802_11 \\
\hline No. of Nodes & 27 \\
\hline Radio Protocol & AODV \\
\hline Value (x) & 500 \\
\hline Value (y) & 500 \\
\hline ns & new Simulator \\
\hline
\end{tabular}

Table 1. Configure and Create Nodes $\mathrm{N}=7$

3. Define color index

4. Define source and destination positions

5. Set up a flow: Flow of a route can be set up by using following channels.

set chan_1 [new \$val(chan)]

set chan_2 [new \$val(chan)]

set chan_3 [new \$val (chan)]

set chan_4 [new \$val (chan)]

6. Request data

7. Execute simulation. 
International Journal of Wireless \& Mobile Networks (IJWMN) Vol. 3, No. 5, October 2011

\section{IV.Simulation Results:}

Following are the real-time snapshots taken for the calculation of throughput .Fig.6, Fig.7 and Fig.8shows transmission of message at different time periods. The green color nodes of the fig.6 indicates one route for the transmission of packets from source to destination...Simultaneously sensing of the nodes along with the transmission of packet takes place.Fig.7 shows state of changing route. Whereas fig.8 shows blue color nodes frorn different route along with the sensing of the nodes. Throughput is the ratio of the total amount of data that reaches a receiver from a sender to the time it takes for the receiver to get the last packet $[13,14,15$, and 16]. It measures of effectiveness of a routing protocol. The throughput values of AODV Protocols for 7 Nodes at Pause time 10s are noted and they are plotted on the different scales to best show the effects of varying throughput of the above routing protocols (Figures $6,7 \& 8$ ). Based on the simulation results, the throughput value of AODV increases initially and reduces when the time increases $[17,18,19]$. The throughput value of AODV slowly increases initially and maintains its value when the time increases.

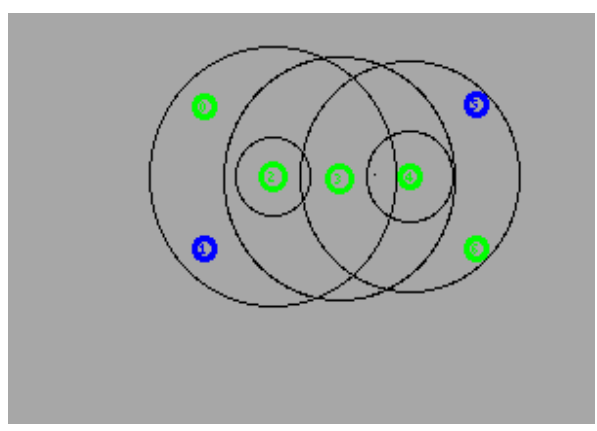

Fig.6. Initially with N0 as a source and N5 destination

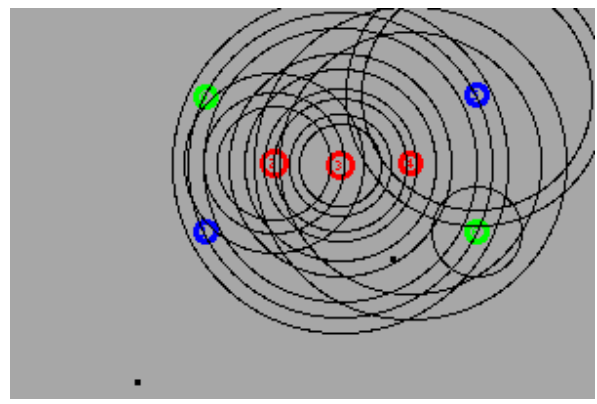

Fig.7. Packet transmission with spectrum sensing

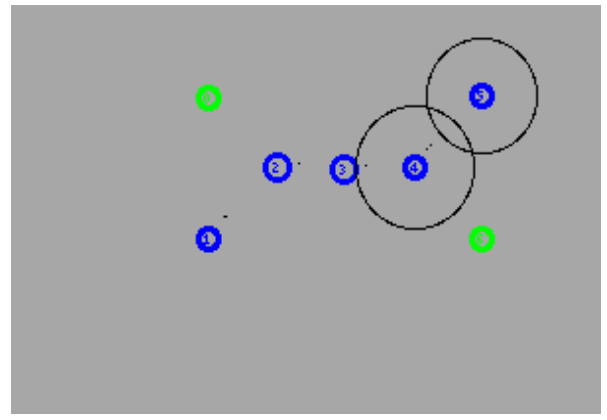

Fig.8. Initially with N1 as a source and N6 destination 
The duration of the periodic spectrum sensing decides, in part, the end-to-end performance a shorter sensing time may result in higher throughput but may affect the transport layer severely if a PU is misdirected.

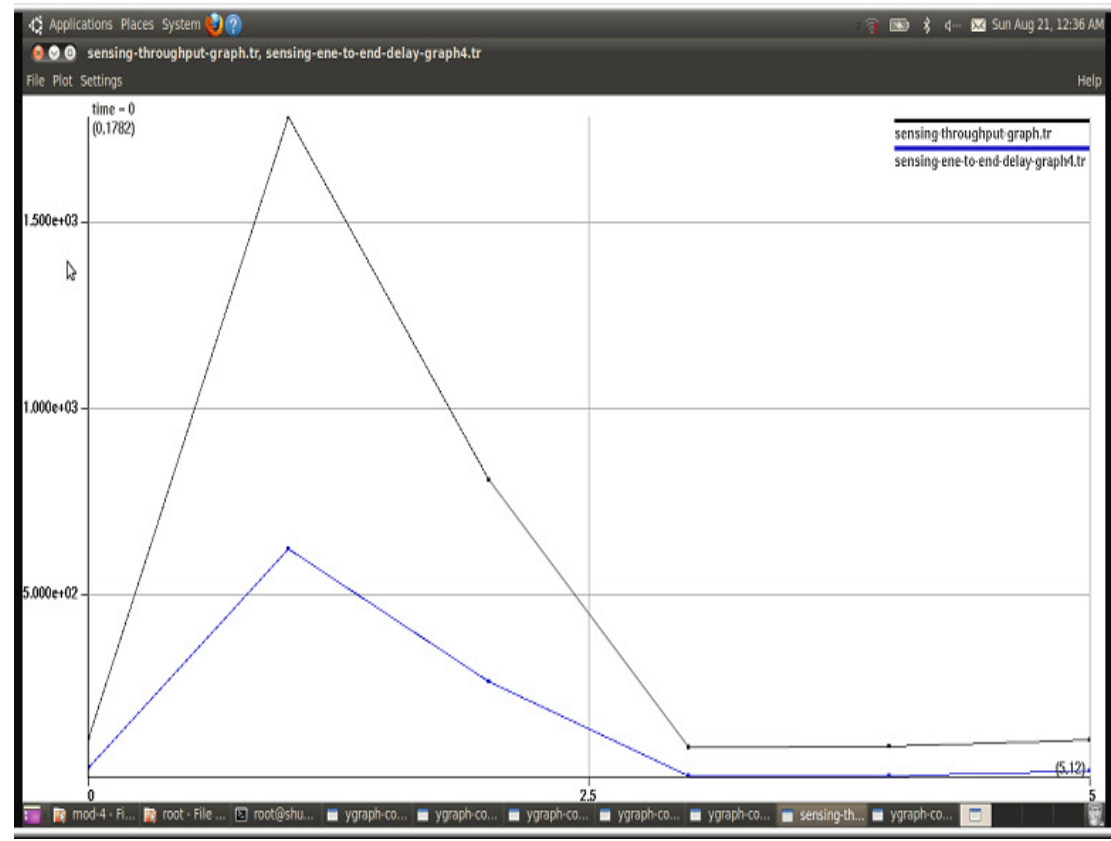

Fig.9.Throughput for CRAHNs with respect to sensing Time with End to End Delay

\section{Conclusion:}

Thus, the sensing scheme needs to be integrated in the design of the transport protocol. Fig.9 shows throughput of a CRAHNs.It clearly shows that the throughput with routing is more than the throughput without routing. This analysis of throughput will be required whenever there is a requirement of the delay calculation in case of handoff mechanism. As during the handoff mechanism during routing process switching delay and node delay occurs and that will be the challenge for the researchers.

\section{Authors}

1. Ms.Shubhangi Mahamuni received her B.E and M.E.degree in Electronics Engineering from Aurangabad University. She is currently working towards the $\mathrm{PhD}$ degree in the department of Electronics, Sardar Patel National Institute of Technology, Surat, India under the guidance of Dr.Vivekanand Mishra (Senior Member IEEE) and Dr.Vijay Wadhai.. Her research interests include Wireless Communications, Cognitive Radio Networks and cross-layer communication protocols, heterogeneous wireless architectures and wireless sensor networks.

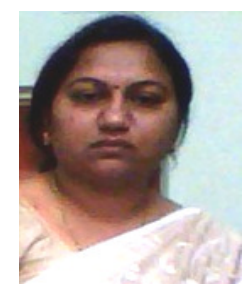


International Journal of Wireless \& Mobile Networks (IJWMN) Vol. 3, No. 5, October 2011

2. Dr.Vivekanand Mishra, working as Associate Professor, at SVNIT, Surat, received his Ph.D. degree from Institute of Technology, B.H.U. India .He was a Research Fellow, Telecom Malaysia Research and Development. He was member in center for photonic research and application (CPRA) and chairman optical communication group (ocg) in multimedia university Malaysia. He has also done extensive research in areas like Analysis design and construction of acoustic tunable filter based on tapered fiber funded by E- Science, Malaysian Government, S-band optical amplifier, Multiple gas sensing using air guiding photonic band gap- fibers. His current research interests include optical communication, Electromagnetic Engineering, Wireless communication. He had 30 International Journal, 14 International Conference Publications and 2 national

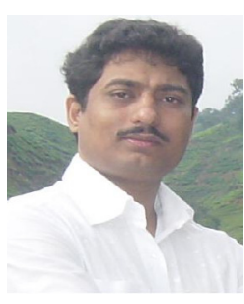

3. Dr.Vijay M.Wadhai, currently working as a Professor and Principal, MITCOE,Pune.He received his Ph.D.degree in Electronics Engineering from Gadge Baba Amravati University; Amravati .His main research is in Cognitive Radio, Wireless Communication and VLSI design. Also he had wide academic experience. He had 25 International Journal, 23 International Conference Publications and 17 national publications.

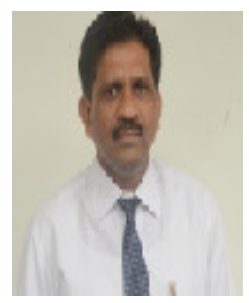

\section{REFERENCES:}

[1] I. Akyildiz, W.Y. Lee, K.R. Chowdhury, CRAHNs: Cognitive Radio Ad HoC Networks, Ad Hoc Networks Journal 7,2009, 810-836.

[2] Tao Lin, Scott F.Midkiff and Jahng S.Park,A framework for Wireless Ad hoc Routing Protcols, IEEE 2003.

[3] Zuraida Binti Abdullah Hani and Mohd. Dani Bin Baba, Designing Routing protocols for Mobile Ad hoc networks,IEEE 2003.

[4] Dhiraj Nitnaware, Ajay Verma, Energy constraint Node cache based routing protocol for Adhoc Network, IJWMN, Feb. 2010.

[5] Mehran Abolhasan, Tadeusz Wysoci, Eryk Dutkiewicz, A review of routing protocols for mobile ad hoc networks, ELSEVIER , 2003.

[6] Changling Liu, Jorg Kaiser, A survey of Mobile Ad Hoc Network Routing Protocols, University of Magdeburg, 2005.

[7] Yih-Chun Hu and David B.Johnson ,Caching Strategies in On-Demand Routing Protocols for Wireless Ad hoc Networks, ACM 2000.

[8] Mehran Abolhasan, Tadeusz Wysocki and Eryk Dutkiewicz, A review of routing protocols for mobile ad hoc networks, Elsevier 2003.

[9] K.R. Chowdhury, I.F. Akyildiz, Cognitive wireless mesh networks with dynamic spectrum access, IEEE Journal of Selected Areas in Communications 26, 2008,168-181.

[10] K.R. Chowdhury, M. Di Felice, Search: a routing protocol for mobile cognitive radio ad-hoc networks, Computer Communication Journal 32, 2009, 1983-1997.

[11] K. Akkaya, M. Younis, A survey on routing protocols for wireless sensor networks, Ad Hoc Networks (Elsevier) 3,2005, 325-349

[12] C.Perkins, Ad hoc on-demand distance vector (AODV) routing, RFC 3561,July 2003

[13] M. Gandetto, C. Regazzoni, Spectrum sensing: a distributed approach for cognitive terminals, IEEE Journal on Selected Areas in Communications 25,2007, 546-557.

[14] Goff, J. Moronski, D.S. Phatak, V. Gupta, Freeze-TCP: a true end-to-end TCP enhancement mechanism for mobile environments, in: Proceedings of the IEEE INFOCOM, Tel-Aviv, Israel, 2000,1537-1545.

[15] L. Hanzo (II.), R. Tafazolli, A survey of QoS routing solutions for mobile ad hoc networks, in: IEEE Communications Surveys and Tutorials, vol. 9 (2), 2007,50-70. 
International Journal of Wireless \& Mobile Networks (IJWMN) Vol. 3, No. 5, October 2011

[16] K. Chandran, S. Raghunathan, S. Venkatesan, R. Prakash, A feedback based scheme for improving TCP performance in ad hoc wireless Networks, IEEE Personal Communications Magazine 8,2001, 34- 39.

[17] K.R. Chowdhury, M.D. Felice, SEARCH: a routing protocol for mobile cognitive radio ad-hoc networks,2008,313-320.

[18] C. Cordeiro, K. Challapali, C-MAC: a cognitive MAC protocol for multi-channel wireless networks, in: Proceedings of the IEEE DySPAN,2007, 147-157.

[19] H.M. El-Sayed,O.Bazon and U.Qureshi and M.Jaseemuddin Performance Evaluation of TCP in Mobile Ad Hoc Networks.

[20] Network simulator version $2.34 \mathrm{http} / / / \mathrm{www}$.isi.edu/nsnam/ns/.

L. Cao, H. Zheng, Distributed spectrum allocation via local bargaining, in: Proceedings of the IEEE Sensor and Ad Hoc Communications and Networks (SECON), 2005, 475-486.

[21] Saiful Azadm, Arafatur Rahman and Farhat Anwar, A Performance comparison of Proactive and Reactive Routing protocols of Mobile Ad hoc Networks(MANET)), Journal of Engineering and Applied Sciences, 2007. 